\title{
Vom Nutzen und Nachteil der Frauen- und Geschlechtergeschichte für die Gender Theorie Oder: Warum Geschichte wichtig ist
}

Regina Wecker

\section{Einleitung}

„Geschichte boomt.“ Noch nie habe es ein so breites Interesse an Geschichte gegeben, stellte Hermann Lübbe, emeritierter Professor für Philosophie und Politische Theorie an der Universität Zürich, kürzlich fest. ${ }^{1}$ Dieses Interesse manifestiere sich in aufwändigen Gebäuderestaurationen, in „Folklore und Heritage-Sucht“ oder im Interesse an serienmäßigen Dokumentarfilmen und Fotoeditionen, Medienspektakeln, Museen, Ausstellungen und Publikationen. Je schneller sich die Gegenwart ändere, umso mehr bräuchte es die Geschichte, die das kompensiere, erklärte Lübbe diesen Boom. Die HistorikerInnen Valentin Groebner, Madeleine Herren, Roger Sablonier und der Soziologe Rudolf Stichweh auf dem Podium und ein Teil der ZuhörerInnen waren skeptisch, sowohl was die Bedeutung der historischen Dimension angeht, als auch, ob sie dieses Interesse bedienen wollen, da es in ihren Augen kaum der Auseinandersetzung mit der Vergangenheit oder dem Verständnis von Gegenwart diene, schon gar nicht in diese „hineinwirke“ oder zu einer Erweiterung verengter Horizonte beitrage. Wer aber das kritische Reflexionspotential der Geschichtswissenschaft mit diesen Zielen nutze und weder nostalgische Verklärung oder Identitätspolitik betreibe, noch das „ach wie gut haben wir es doch heute-Gefühl“ produziere, der riskiere - so der Luzerner Historiker Valentin Groebner - „als Spezialist für schlechte Laune“ dazustehen. Trotz Interessenzuwachs also Bedeutungsverlust - trotz Nostalgie Geschichtslosigkeit?

Ähnliche Fragen wirft die Stellung der Geschichte in interdisziplinären Zusammenhängen auf. Dass die historische Dimension in den Gender Studies derzeit an Bedeutung verliere, obwohl auch hier weiterhin Beispiele aus der Geschlechtergeschichte zum

I Podiumsdiskussion „Wieviel Geschichte braucht die moderne Wissensgesellschaft?“, moderiert von Susanna Burghartz anlässlich der „Ersten Schweizerischen Geschichtstage“, 15.-17.3.2007 in Bern. 
Argumentationsrepertoire gehören, wird in verschiedenen Zusammenhängen festgehalten. So habe - wie Claudia Opitz kürzlich meinte - der „linguistic turn“ zu einer gewissen "Geschichtsvergessenheit" geführt oder zumindest dazu beigetragen, dass die historische Dimension vieler Phänomene und Entwicklungen in den Hintergrund gedrängt werde ${ }^{2}$. Judith Bennett führt in ihrem kürzlich erschienenen Buch „History Matters" aus, dass neuere feministische Theorien weitgehend ohne Kenntnis der Geschichte auszukommen versuchen, und dass Frauengeschichte, einst „critical battlefront of the feminist struggle" heute als irrelevant erachtet würde. ${ }^{3}$ Auch bei den Überblickswerken, Lexika und Handbüchern stünden geschlechtergeschichtliche Aspekte eher im Hintergrund - so Bea Lundt ${ }^{4}$ - mit Blick auf das Metzler Lexikon "Gender Studies“ oder Hanna Hacker für das Handbuch der Gender Theorien „Gender@Wissen“.5

Allerdings stagniere auch die Rezeption von Forschungsergebnissen der Frauen- und Geschlechtergeschichte seitens der Geschichtswissenschaft. Karin Hausen schließt daraus, dass eine Allgemeine Geschichte, die die Geschlechtergeschichte einschließe, nicht möglich und auch nicht sinnvoll sei, und man daher die Forderung nach der "Einheit der Geschichte“ aufgeben und die reale Nicht-Einheit der Geschichte akzeptieren sollte. ${ }^{6}$ Während Judith Bennet das Aufgehen der Frauengeschichte in der Geschlechtergeschichte als Gefahr in Bezug auf ihre praktische Bedeutung und auf die Bedeutung für die historischen Forschung einschätzt, ${ }^{7}$ gehen andere davon aus, dass die Geschichtswissenschaft der Herausforderungen der Geschlechtergeschichte bald einmal nicht mehr bedürfe, da sie bereits zum selbstverständlichen Bestandteil der Geschichtswissenschaft geworden sei. ${ }^{8}$

Ziel meines Beitrags ist es, diesen Vorstellungen, Entwicklungen und Phänomenen nachzugehen und danach zu fragen, ob und wie sie zusammenhängen: die angebliche ,Geschichtslosigkeit` unserer Zeit und das breite Interesse an allem, was alt ist; die

2 Claudia Opitz, Nach der Gender-Forschung ist vor der Gender-Forschung. Plädoyer für die historische Perspektive in der Geschlechterforschung, Vortrag an der Tagung der Sektion Frauen- und Geschlechterforschung der Deutschen Gesellschaft für Erziehungswissenschaft „Was kommt nach der ,Genderforschung? Ein interdisziplinäres Gespräch“, 8.-10.6.2007 in Marburg.

3 Judith M. Bennett, History Matters. Patriarchy and the Challenge of Feminism, Philadelphia 2006, 2.

4 Bea Lundt, Einleitung in den Schwerpunkt. Von der sozialen Bewegung zu professionellen Geschlechterforschung, in: Historische Mitteilungen, 19 (2006), 1-13, 5.

5 Hanna Hacker, Rezension von Christina von Braun u. Inge Stephan Hg., Gender@Wissen. Ein Handbuch der Gender-Theorien, Köln 2005, in: L'HOMME. Z. F. G., 18, 1 (2007), 166-169.

6 Karin Hausen, Die Nicht-Einheit der Geschichte als historiographische Herausforderung. Zur historiographischen Relevanz und Anstößigkeit der Geschlechtergeschichte, in: Hans Medick u. AnneCharlotte Trepp Hg., Geschlechtergeschichte und Allgemeine Geschichte. Herausforderungen und Perspektiven, Göttingen 1998, 15-55.

7 Bennett, History, wie Anm. 3, 9ff.

8 Dorothea Wierling, Keine Frauengeschichte mehr nach dem Jahr 2000, in: Jarausch Konrad, Jörn Rüsen u. Hans Schleier Hg., Geschichtswissenschaft vor 2000, Hagen 1994, 440-456. 
Rezeptionssperre gegenüber Forschungsergebnissen der Geschlechtergeschichte; der Bedeutungsverlust der historischen Perspektive in den Gender Studies sowie die Beziehung der Frauengeschichte zur Geschlechtergeschichte. Ich frage dabei nach dem Nutzen der historischen Dimension, insbesondere dem Nutzen einer langfristigen historischen Perspektive und werde dabei auch Bedeutung und Konzeptualisierung von Bruch und Kontinuität ansprechen.

Den Ausgangspunkt bilden einige Bemerkungen zur Entstehung der wissenschaftlichen Disziplin Geschichte und ihrer Kritiker im 19. Jahrhundert wobei ich auf den Prozess der Verdrängung von Historikerinnen fokussiere (II), gefolgt von der Darstellung der Entwicklung der Frauengeschichte und jener der Geschlechtergeschichte (III und IV). Im letzten Teil werde ich am Beispiel der Geschichte der Eugenik die angesprochenen Entwicklungen und Tendenzen nochmals konkretisieren (V und VI).

Der Titel, in dem Friedrich Nietzsches „Unzeitgemäße Betrachtungen“9 unschwer zu erkennen sind, soll nicht nur sprichwörtlich dafür stehen, dass „jedes Ding zwei Seiten hat" ${ }^{10}$ und somit auch die Geschichte mal nützlich und mal hinderlich sein kann, sondern eine Ambivalenz zum Ausdruck bringen, welche die Geschichte der Geschichtswissenschaft und die Bedeutung der Geschlechtergeschichte innerhalb der Geschichtswissenschaft und der Gender Studies grundlegend durchzieht und letztlich auch mit Ambivalenzen moderner Wissenschaft generell zu tun hat.

\section{Geschichte als wissenschaftliche Disziplin und der Ausschluss der Frauen}

In „Vom Nutzen und Nachteil der Historie für das Leben“ setzt sich Friedrich Nietzsche mit der Bedeutung von Geschichte auseinander. Unzeitgemäß nennt er seine Auseinandersetzung, weil er „etwas, worauf die Zeit mit Recht stolz ist, ihre historische Bildung, hier einmal als Schaden, Gebresten und Mangel der Zeit zu verstehen" versuchte. ${ }^{11}$ Die Fähigkeit, sich zu erinnern, sei Voraussetzung jeder Kultur, die Fähigkeit zu vergessen aber, „das Vergessen-können“, sei ebenso wichtig. ${ }^{12}$

„[J]eder Mensch und jedes Volk braucht je nach seinen Zielen, Kräften und Nöthen eine gewisse Kenntniss der Vergangenheit ..."13 Aber nur, wenn sie im Dienste der

9 Friedrich Nietzsche, Vom Nutzen und Nachtheil der Historie für das Leben (1874). Unzeitgemäße Betrachtungen. Zweites Stück, in: ders., Die Geburt der Tragödie. Unzeitgemäße Betrachtungen IIV. Nachgelassene Schriften 1870-1873. Kritische Studienausgabe hg. von Giorgio Colli u. Mazzino Montinari, München/Berlin/New York 1988, 243-334.

IO Katrin Meyer, Ästhetik der Historie. Friedrich Nietzsches „Vom Nutzen und Nachteil der Historie für das Leben“, Würzburg 1998, 1.

II Nietzsche, Nutzen, wie Anm. 9, 246.

I2 Nietzsche, Nutzen, wie Anm. 9, 250.

I3 Nietzsche, Nutzen, wie Anm. 9, 271. 
Zukunft und der Gegenwart stehe, habe Erinnerung, habe Historie ihre Berechtigung. "Nur soweit die Historie dem Leben dient, wollen wir ihr dienen. ${ }^{\text {" }}$ "Im vierten Kapitel der Schrift warnt er vor der Zerstörung dieser Verbindung von Leben und Historie. Das zerstörende "Gestirn" sieht er in der Forderung, dass die Historie Wissenschaft zu sein habe. „Ein solches unüberschaubares Schauspiel sah noch kein Geschlecht, wie es jetzt die Wissenschaft des universalen Werdens, die Historie, zeigt ..." ${ }^{15}$

Bereits in seiner Einleitung zu den zweiten „Unzeitgemäßen Betrachtungen“ hatte Nietzsche festgehalten, dass er sich gegen eine mächtige historische "Zeitrichtung“ wende, wie sie „bekanntlich seit zwei Menschaltern unter den Deutschen namentlich zu bemerken ist ${ }^{\text {". }}{ }^{16}$ Nietzsches Kritik an der Historie als Wissenschaft, seine ,HistorismusKritik', muss durch diese inhaltliche und zeitliche Bestimmung sehr weit gefasst werden: Inhaltlich kann sie auf verschiedene Entwicklungen, Phänomene, Ausprägungen der Geschichtswissenschaft bezogen werden: den Positivismus, die empirische Geschichtswissenschaft, den universitären Forschungsbetrieb und Rationalitäts- und Objektivitätsforderungen. ${ }^{17}$ Sie bezieht sich auf den Prozess der Verwissenschaftlichung der Historie einerseits und andererseits auf den Prozess der Historisierung der Wissenschaften. ${ }^{18}$ Zeitlich geht sie damit über die eigentliche Zeit des Historismus und die des 19. Jahrhunderts hinaus. Polemisch hatte Nietzsche hier eine Kritik vorweggenommen, die auch später gegenüber einer ,sich im weltfremden Forschungspositivismus verlierenden Wissenschaft ${ }^{119}$ ins Feld geführt wurde, ohne dass er den Begriff Historismus verwendet: Geschichte als Wissenschaft mache das Wissen um Ereignisse, Entwicklungen und Personen der Vergangenheit zum Selbstzweck, die Historisierung von Wissen und Wissenschaft komme dem Verzicht auf ein eigenes wertendes Urteil gleich. Nietzsches Kritik am Objektivitätsideal der (Geschichts-)Wissenschaft ist demnach keine, die bloß einen oberflächlichen Nachteil der Historie einklagt. Der Nachteil - und auch der Nutzen - der Geschichtsschreibung hängen seiner Meinung nach grundsätzlich davon ab, wie Geschichte als Wissenschaft konstruiert wird, welchen Interessen sie dient, welchen Effekt sie für die modernen Subjekte hat und wie sich damit Machtverhältnisse reproduzieren. Gerade diese praktisch-politischen Dimensionen sollten - seiner Meinung nach - aber in der modernen Geschichtswissenschaft ausgeklammert werden. ${ }^{20}$ Die Geschichtsschreibung und die Bedeutung der historischen Dimension kann Nietzsche also gleichzeitig legitimieren und vernichtend kritisieren. Wenn die TeilnehmerInnen der eingangs erwähnten Podiumsdiskussion sich dagegen

I4 Nietzsche, Nutzen, wie Anm. 9, 245.

I5 Nietzsche, Nutzen, wie Anm. 9, 272.

I6 Nietzsche, Nutzen, wie Anm. 9, 246.

17 Vgl. Meyer, Ästhetik, wie Anm. 10, 67.

I8 Vgl. Meyer, Ästhetik, wie Anm. 10, 68.

I9 Friedrich Jaeger u. Jörn Rüsen, Geschichte des Historismus, München 1992, 103.

20 Ich danke Katrin Meyer für viele wichtige Hinweise zu Nietzsches Geschichtsverständnis, für die kritische Lektüre meines Textes und ihre anregenden Bemerkungen zu Fragen der Gender Studies. 
wehren, als Produzenten von Abstammungslehren missbraucht zu werden, und sich weigern, historisches Wissen als Kompensation für eine ,garstige Gegenwart' zur Verfügung zu stellen, dann könnten sie sich dabei durchaus auf Nietzsche berufen. Die Vorstellung, die am Podium implizit mitschwang, dass allein die universitäre HistorikerInnen wüssten, wo die ,Identitätspflege' beginne und die kritische Auseinandersetzung anfange, hätte wohl kaum Unterstützung gefunden.

Nietzsche erwähnt den wohl wichtigsten Protagonisten der neuen Geschichtswissenschaft, den deutschen Historiker Leopold von Ranke (1795-1886) eher beiläufig. Er kritisiert aber die Art und Weise, wie Geschichte sich zu einer universitären Disziplin neuer Prägung stilisierte. Dieser Prozess wird sehr eng mit Ranke verbunden, obwohl Diskussionen um wissenschaftliche Standards, aber auch um das Verhältnis von Literatur und Geschichte bereits in der Historiographie und Geschichtsphilosophie der Aufklärung, insbesondere bei Johann Christoph Gatterer zu finden sind. ${ }^{21}$ Seine Gründung des Historischen Instituts (1765) hatte die Verwissenschaftlichung der Geschichte institutionell vorangetrieben.

Von Ranke gingen indes die methodisch und darstellerisch bedeutendsten Impulse aus. Er hat Geschichte als moderne Wissenschaft etabliert, die ihre (belegbaren) Kenntnisse aus quellenkritischem Umgang mit Materialien und Objekten zog und damit beanspruchen konnte, ,empirisch fundierte historische Richtigkeiten zu produzieren “.22 An der bisherigen Geschichtsschreibung hatte Ranke kritisiert, dass sie sich auf Überlieferung, Mythen und Erzählungen gestützt habe, die Objektivität vermissen ließen. Historische Kenntnisse resultieren für ihn nur aus dem Studium von Quellen:

Ich sehe die Zeit kommen, wo wir die neuere Geschichte nicht mehr auf die Berichte, selbst nicht der gleichzeitigen Historiker, außer insoweit ihnen eine originale Kenntnis beiwohnte, geschweige denn auf die weiter abgeleiteten Bearbeitungen zu gründen haben, sondern aus den Relationen der Augenzeugen und den ächtesten unmittelbarsten Urkunden aufbauen werden. ${ }^{23}$

Auch wenn Ranke - wie Helmut $\mathrm{Puff}^{24}$ zeigt - in seinen eigenen Darstellungen durchaus nicht so radikal mit der Erzähltradition bricht, wie gemeinhin angenommen wird, insbesondere dann nicht, wenn man „Wissenschaftsgeschichte nicht als Ideengeschichte, sondern als Textgeschichte begreift" ${ }^{25}$ verweisen seine methodisch-programmatischen

2I Johann Christoph Gatterer, Von der Evidenz in der Geschichtskunde (1767), in: Allgemeine Welthistorie in England, hg. von Friedrich Eberhard Boysen, Halle 1767, 466-478.

22 Jaeger/Rüsen, Geschichte, wie Anm. 19, 81.

23 Leopold von Ranke, Deutsche Geschichte im Zeitalter der Reformation, hg. von Paul Joachimsen, München 1925, Bd. 1, 6.

24 Helmut Puff, Männerliebe, Sprache, akademische Maskulinität. Leopold von Ranke und Jacob Burckhardt im Zwiegespräch, in: L'HOMME. Z. F. G., 14, 2 (2003), 298-316, 314.

25 Puff, Männerliebe, wie Anm. 24, 313. 
Ausführungen und Forderungen auf neue Ansprüche an die Geschichtsschreibung; Ansprüche, die dem Nietzscheschen Sinn vom Nutzen der Historie für die Gegenwart widersprechen.

Zwar hat Ranke gefordert, Geschichte müsse sowohl Wissenschaft als auch Kunst sein, dabei aber die Priorität der Forschung immer wieder betont. ${ }^{26}$ Eine Zusammenführung von Literatur, Philosophie und Geschichte war nur unter dem Primat der Geschichtswissenschaft möglich. Deutlich wird das an seiner Einstellung zu Sir Walter Scott, dem schottischen Verfasser äußerst erfolgreicher historischer Romane. Er bewunderte dessen Darstellungskraft, die die Vergangenheit zum Leben erweckt und sie dem Leser damit näher gebracht hätte als manche Archivstudie, betonte aber, dass die historische Wahrheit nicht entstellt werden und die künstlerische Darstellung dem Wahrheitsanspruch nicht im Wege stehen dürfe. Das aber wirft er Scott vor. ${ }^{27}$

Ranke führte das Seminar als Vermittlungs- oder Unterrichtsform neben der Vorlesung ein. Das Seminar, für das er ein Handvoll Studenten zu sich nach Hause einlud, ersetzte bald die Vorlesung als den wichtigsten Ort der Wissensvermittlung. Anders als in der Vorlesung, die eine öffentliche Veranstaltung war, blieb das Seminar bis zur Zulassung von Frauen als Studentinnen Männern vorbehalten. ${ }^{28}$ Die Einführung des Seminars war allerdings nur ein Teil der ,Disziplinierung' der Geschichte und auch nur ein Aspekt des gleichzeitigen Ausschlusses von Frauen als Subjekte und Objekte der Geschichte. Frauen wurden nicht nur ausgeschlossen, indem man ihnen den Zutritt zu den Seminaren und Archiven verwehrte, so dass sie die nun erforderlichen wissenschaftlichen Kenntnisse nicht erwerben konnten, sondern auch dadurch, dass ihre Werke aufgrund des Genres, ihres Schreibstils und ihrer Thematik zunehmend nicht als Geschichtsschreibung galten. Sie wurden durch die Etablierung der ,Professionellen' zu ,Amateurinnen'. Bonnie G. Smith bezeichnet die historischen Darstellungen dieser Amateurinnen als „negative springboard“, von dem die Historiker à la Ranke ihre Ausführungen als die wertvolleren oder einzig wahren historischen Werke über die Geschichte auf den wissenschaftlichen Markt bringen konnten. ${ }^{29}$ Zur Gruppe der Amateurinnen zählt Smith Frauen wie Germaine de Ställ, Stephanie Félicité de Genlis, Victorine de Chastenay, Lucy Aikin, Lydia Maria Child, Christina Belgiojoso, Mercy Otis Warren und Johanna Schopenhauer; Frauen, die uns bezeichnenderweise - wenn überhaupt - als Schriftstellerinnen bekannt sind.

26 Hans Schleier, Geschichtstheorie und Geschichtsschreibung bei Leopold von Ranke, in: Wolfgang J. Mommsen Hg., Leopold von Ranke und die moderne Geschichtswissenschaft, Stuttgart 1988, 115$130,115$.

27 Hermann von der Dunk, Die historische Darstellung bei Ranke. Literatur und Wissenschaft, in: Mommsen, Leopold von Ranke, wie Anm. 26, 131-165, 143.

28 In Preußen erfolgte die Zulassung von Frauen zum Studium 1908.

29 Bonnie G. Smith, The Gender of History. Men, Women, and Historical Practice, Cambridge 1998, 37. 
Angelika Epple erweitert dieses Bild und die Frage nach den Historikerinnen in ihrer interessanten Analyse der um 1800 entstandenen Erzählungen von Frauen aus dem deutschen Sprachraum, deren Arbeiten sie mit dem Begriff „empfindsame Geschichtsschreibung" charakterisiert. ${ }^{30}$ Epple führt Beispiele historischer Erzählungen von Frauen an, die mehrheitlich Biographisches oder Autobiographisches verarbeitet haben. Diese Werke sind in erster Linie historisch, nicht literarisch: Sie verfolgen einen historischen Modus des Erzählens, der sich auf „tatsächliche Erfahrung“ stützt, während die Fiktion, der literarische Modus, über die Darstellung „möglicher Erfahrung“ Plausibilität erlangt. ${ }^{31}$ Sie vermitteln, wie Epple ausführt, individuelle und gesellschaftliche Zeitdeutung und grenzen sich durch diesen „historischen Pakt“ von Literatur ab.

Ulrike Weckel hat in ihrer Analyse der ersten deutschen Frauenzeitschriften im späten 18. Jahrhundert ${ }^{32}$ gezeigt, dass biographische Geschichtsschreibung von Frauen im 17. und 18. Jahrhundert - vor der Entstehung der deutschen „Historischen Schule“ Rankes - Tradition hatte. Geschichte galt durchaus als,vertretbare Beschäftigung' für Frauen, es schien auch für sie möglich und wünschenswert, aus der Geschichte Lehren für die Gegenwart zu ziehen. Die Autorinnen der deutschen Frauenzeitschriften des 18. Jahrhunderts gingen vor dem Hintergrund des Topos der ,historia magistra vitae davon aus, dass auch die Geschichte „eine von den nützlichen Wissenschaften“ ist, „von welchen sich das weibliche Geschlecht nicht ganz ausschließen soll“, wie eine von ihnen 1792 formulierte. ${ }^{33}$

Warum aber sind diese „Konkurrenzunternehmen zur akademischen Deutung der Vergangenheit “34 später nicht mehr als historische Darstellungen erfasst worden? Die Frage enthält bereits einen Teil der Antwort: Gegenüber dem Postulat, dass Geschichte Wissenschaft sei, ihre Legitimation über wissenschaftliche Kriterien erlangen musste, und Wissenschaft und der wissenschaftliche Wahrheitsbeweis zudem ganz allgemein zum Königsweg wurden, hatten nichtwissenschaftliche ,Konkurrenzunternehmen“, auch die von Männern, einen schweren Stand. Frauen aber hatten zusätzliche Schwierigkeiten: Sie mussten legitimieren, warum sie sich gerade als Frauen bestimmten Themen, zum Beispiel der politischen Geschichte, widmeten. Behandelten Frauen aber Themen,

30 Angelika Epple, Empfindsame Geschichtsschreibung. Eine Geschlechtergeschichte der Historiographie zwischen Aufklärung und Historismus, Köln u. a. 2003; vgl. auch die Rezension von Gianna Pomata in diesem Band. Der Gattungsbegriff lehnt sich an die Poetik der Empfindsamkeit an und postuliert ein weiblich konnotiertes Erzählmuster, das auch in den historischen Darstellungen die Unmittelbarkeit des Gefühlsausdrucks sucht.

3I Angelika Epple, Historiographiegeschichte als Diskursanalyse und Analytik der Macht. Eine Neubestimmung der Geschichtsschreibung unter den Bedingungen der Geschlechtergeschichte, in: L'HOMME. Z. F. G., 15, 1 (2004), 77-96, 87f.

32 Ulrike Weckel, Zwischen Häuslichkeit und Öffentlichkeit. Die ersten deutschen Frauenzeitschriften im späten 18. Jahrhundert und ihr Publikum, Tübingen 1998, 532ff.

33 Weckel, Häuslichkeit, wie Anm. 32, 535.

34 Epple, Geschichtsschreibung, wie Anm. 30, 3. 
des ,alltäglichen Lebens', Biographisches, so zählten ihre Werke wegen der Vorstellung, dass Geschichte nun vor allem Geschichte des Politischen wäre, ebenfalls nicht zu den historischen. Die Trennung zwischen öffentlicher und privater Sphäre und die strikte Zuordnung des Politischen zum Öffentlichen, die die Geschichtswissenschaft des 19. Jahrhunderts konstituierten, tat also zusätzlich ihre Wirkung. In jedem Fall aber diskreditierte Autorinnen ihr empfindsamer Stil, der nach Gatterer dem Roman zuzurechnen ist und nicht dem historischen Werk. Der „critische“ Leser muss - so Gatterer - im Gegensatz zum „empfindsamen“ Leser, für den die evidente Erzählung ausreichend ist, durch Beweise überzeugt werden. ${ }^{35}$ Geschichtsschreibung folgt nun anderen Prämissen als Literatur. ${ }^{36}$

Die ,moderne' Geschichtsschreibung musste auch mit der Vorstellung von der Geschichte als Lehrmeisterin brechen. Zum einen sollte jede Epoche, für sich selbst stehen und um ihrer selbst willen untersucht werden und nicht in Hinblick auf die Gegenwart: "nicht auf diese gegenwärtige Vergangenheit, sondern auf die Vergangenheit als die Vergangene ist es dem Menschen gemäß sich zu richten ". ${ }^{37}$ Zum anderen zeichnet sich die Moderne gerade durch ihre Vorstellung vom Bruch mit der Vergangenheit aus, durch die Vorstellung, etwas insgesamt Neues zu schaffen und zu sein..$^{38}$ Der Bruch wird zu einem wesentlichen Merkmal einer an Fortschritt orientierten Darstellung von Vergangenheit. Damit wird die Hoffnung, direkt aus der Geschichte lernen zu können, zumindest limitiert.

Die Forderung nach klarer Trennung, Aufteilung und Abgrenzung von Disziplinen und die Vorstellung der Trennung „wissenschaftlich - unwissenschaftlich“ ist nicht auf die Geschichtswissenschaft beschränkt, vielmehr ist sie ein allgemeines Kennzeichen der Entwicklung der Wissenschaften im 19. Jahrhundert und wird darüber hinaus insgesamt zum Kennzeichen der Moderne. Politische und wirtschaftliche Maßnahmen, von der Landwirtschaftspolitik bis zur Geburten- und Bevölkerungspolitik werden nun wissenschaftlich legitimiert. Michel Foucault hat mit dem Begriff der „Biopolitik“ die neuen Strategien und Taktiken des Staates im Umgang mit dem Lebenden gefasst und das Überschreiten der „biologischen Modernitätsschwelle“ einer Gesellschaft dort festgestellt, „wo es in ihren politischen Strategien um die Existenz der Gattung selber geht. ${ }^{\text {"39 }}$ Die Ende des 19. Jahrhunderts als neue Wissenschaft begründete Eugenik oder Rassenhygiene fördert und legitimiert dieses Überschreiten, indem sie für sich in

35 Der Gegensatz von Verstand und Gefühl ist „highly gendered“.

36 Epple, Geschichtsschreibung, wie Anm. 30, 100, bezieht sich auf Johann Christoph Gatterer, Evidenz, wie Anm. 21, 471.

37 Leopold von Ranke, Tagebücher (1814), hg. von Walther Peter Fuchs, München 1964, 109.

$38 \mathrm{Zu}$ Kriterien und Datierung vgl. Zygmunt Bauman, Moderne und Ambivalenz. Das Ende der Eindeutigkeit, Hamburg 2005, 14, Anm. 1.

39 Michel Foucault, Der Wille zum Wissen. Sexualität und Wahrheit 1, Frankfurt a. Main 1997, $170 f$. 
Anspruch nimmt, zwischen gutem und schlechtem Erbgut unterscheiden zu können und die Förderung des einen und die Eliminierung des anderen fordern zu müssen. So hält der Soziologe Zygmunt Bauman fest, dass sich die Moderne „der Fragmentierung der Welt als ihrer bedeutendsten Leistung " rühmt. ${ }^{40}$ Ziel der Fragmentierung ist, Eindeutigkeit zu schaffen. Dies - so Bauman - sei der Anspruch der Moderne, ein Anspruch, an dem sie notgedrungen scheitern müsse. Gerade die Vorstellung, Ambivalenzen ausschalten zu müssen und Entwicklungen in Kategorien wie Fortschritt und Rückschritt eindeutig fassen zu können, schaffe immer neue Ambivalenzen. Bauman verdeutlicht das auch am Beispiel der Eugenik, die er als typisches „Projekt der Moderne" sieht. Ich werde darauf zurückkommen.

Frauen galten also aufgrund ihres Geschlechtscharakters, der fehlenden Autonomie, der Verhaftung im häuslichen, privaten Bereich und der neuen Funktion von Geschichte nicht mehr als ernstzunehmende Historikerinnen. ${ }^{41}$ In der ,modernen Geschichtswissenschaft wird Eindeutigkeit und Wissenschaftlichkeit - so zunächst mein Fazit für diese Epoche der Wissenschaftsgeschichte des Fachs - durch den Ausschluss der Amateurinnen hergestellt.

\section{Frauen- und Geschlechtergeschichte und der ,Kern' der Geschichtswissenschaft}

Als in den 1960er und 70er Jahren die Frauengeschichte den Ausschluss der Frauen aus der Geschichtswissenschaft und die Tatsache, dass sich Konzeptionen, Methoden und Relevanztopoi auf die männlichen Akteure und die ,männlichen' Sphären beschränkten, zu kritisieren begann und - zunächst im englischsprachigen Raum - den Einschluss der Geschichte der Frauen und der weiblich konnotierten Lebensbereiche in historische Analysen forderte, hatten ihre Protagonistinnen das Gefühl, etwas völlig neues ,erfunden ‘ zu haben und sahen sich keinesfalls als Nachfolgerinnen der ,Amateurinnen“ ${ }^{42}$

Inzwischen hatte sich allerdings die Geschichtsschreibung von den Ordnungskriterien des 19. Jahrhunderts weit entfernt. So hatte die Sozialgeschichte der 1960er Jahre zu einer Abwendung von politikzentrierten Ansätzen geführt und dabei auch im deutschsprachigen Raum die Ansätze der Annales wieder aufgenommen. Sowohl der Gegenstandsbereich als auch die Darstellungsmethoden hatten sich verändert. Der traditionell überlieferte Kanon des Faches Geschichte, der für lange Zeit sowohl von festen Gattungsgrenzen und einem festen Gegenstandsbereich als auch von einem festen Wahrheitsbegriff ausgegangen war, war längst erfolgreich infrage gestellt worden -

\footnotetext{
40 Bauman, Moderne, wie Anm. 38, 29.

4I Es sind diese vielfältigen Überschneidungen und Verbindungen die das Geschlechterverhältnis beeinflussen (intersectionality).

42 "We invented it“, Smith, Gender, wie Anm. 29, $37 f$.
} 
nicht nur von der Frauengeschichte. So führt der Historiker und Literaturwissenschaftler Hayden White, der Geschichtsschreibung erstmals mit Kategorien der Literaturtheorie analysierte und damit die Debatte um postmoderne beziehungsweise poststrukturalistische Ansätze in der Geschichtswissenschaft auslöste, den „desolaten Zustand“ der Disziplin noch 1978 darauf zurück, dass ,sie ihre Ursprünge in der literarischen Einbildungskraft aus dem Blick verloren hat, weil sie wissenschaftlich und objektiv erscheinen möchte. ${ }^{\text {" } 33}$ Mit der Forderung, Geschichtsschreibung wieder enger an ihre „literarische Grundlage“ zu binden, fordert White nichts Geringeres als eine Verschiebung der Gattungsgrenzen, wie sie sich seit der Mitte des 18. Jahrhunderts etabliert hatten. Diese galten nicht etwa als in einem bestimmten historischen Kontext entwickelte Merkmale, sondern als unhintergehbare Kriterien von Geschichtswissenschaft, ja von Fortschritt und Moderne insgesamt.

White bewegte sich mit seinen Forderungen nahe an der Vorstellung des „linguistic turn", dass die Erzählung nicht nur die Fakten strukturiert und damit interpretiert, sondern dass Sprache auch Realität konstruiert. Allerdings sieht sich White damit auch in der Tradition der zeitgenössischen Kritiker von Ranke, die - wie etwa Johann Gustav Droysen und Nietzsche - dem „Mythos der Objektivität“ und „der scheinbar plausiblen Forderung nach strenger Wissenschaftlichkeit der Geschichtsschreibung" und der Selbstevidenz der „Fakten“ widersprochen hatten. ${ }^{44}$ White folgt zudem Nietzsche in Bezug auf eine "nützliche“, das heißt ästhetisch kreative und lebendige Geschichtsschreibung. In der deutschsprachigen Geschichtsschreibung hat White heftige Debatten ausgelöst und auch spätere Ansätze der Diskurstheorie wurden hier, wie Franz Eder beobachtet, „,vor allem von den Rändern des geschichtswissenschaftlichen Feldes ... getragen. “45 $\mathrm{Zu}$ diesen Rändern gehörte zweifellos die Frauen- und Geschlechtergeschichte.

Die Frauengeschichte prallte ähnlich hart wie Whites Konzeptionen auf die Regeln und Gegebenheiten, die trotz aller Kritik am Historismus seit dem 19. Jahrhundert das Selbstverständnis und den Kern der historischen Wissenschaft bildeten: die Forderung nach einer bestimmten Form von Objektivität und die Bedeutung, die man den Quellen beimaß. Ein Teil der Schwierigkeiten, die die Frauengeschichte innerhalb der Geschichtswissenschaft in ihren Anfängen hatte, war damit verbunden, dass sie die Ranke'schen Anforderungen nicht anerkennen konnte. Sie war nicht auf die Vergangenheit als das Vergangene gerichtet, sie hatte ein politisches Ziel, sie war nicht

43 Haydn White, Der historische Text als literarisches Kunstwerk, in: ders., Auch Klio dichtet, oder Die Fiktion des Faktischen. Studien zur Tropologie des historischen Diskurses, Stuttgart 1986, 101-122, 122 (Orig. Baltimore/London 1978). Der „desolate Zustand“ bezieht sich insbesondere auf die Dominanz der Struktur- und Sozialgeschichte.

44 Hayden White, Interpretation und Geschichte, in: ders., Klio, wie Anm. 43, 64-100, 66f.

45 Peter Schöttler, Wer hat Angst vor dem „linguistic turn“?, in: Geschichte und Gesellschaft, 23, 1 (1997), 134-151; Franz X. Eder, Historische Diskurse und ihre Analyse - eine Einleitung, in: ders. Hg., Historische Diskursanalysen. Genealogie, Theorie, Anwendungen, Wiesbaden 2006, 9-26, 9. 
,objektiv' im Rankeschen Sinn und - es fehlten die Quellen. Bis in die 1980er Jahre wurde von den ,Gegnern' die Unmöglichkeit, Frauengeschichte zu betreiben, immer wieder damit begründet, dass es nicht ausreichend Quellen von Frauen und über Frauen gäbe. In dieser Vorstellung verbanden sich die Frauen ausschließende Unterrichtsform Rankes und dessen inhaltliche Bewertung der Quellen. Quellen waren zwar „Prinzessinnen“, wie es Ranke einst ausgedrückt hatte, ${ }^{46}$ aber in den ,wichtigen` Quellen kamen Frauen nicht vor. In diesem Kontext entsteht der Eindruck, dass Rankes Einfluss bestehen blieb - trotz aller Kritik, gerade weil man seinen Einfluss nicht mehr als solchen, sondern als das Wesen der Geschichtswissenschaft wahrnahm und diese Wahrnehmung gab der Frauen- und Geschlechtergeschichte den Anstrich der Unwichtigkeit und Unwissenschaftlichkeit. Daran änderte der Siegeszug der Sozial- und Wirtschaftsgeschichte im deutschsprachigen Raum seit der Mitte der 1970er Jahre zunächst nichts. Auch sie untersuchte mit wenigen Ausnahmen Prozesse und Strukturen, ohne dabei nach Geschlechtern differenzierte Kategorien anzuwenden, oder konzentrierte sich auf Bereiche, in denen männliches Verhalten im Mittelpunkt stand. Frauen blieben in Theorie und Begrifflichkeit ausgeklammert, ohne dass dieser Ausschluss als solcher reflektiert oder deklariert wurde.

Im englischsprachigen Raum gelang es zuerst, Frauengeschichte als wissenschaftliches Fach zu etablieren und mit neuen Forschungsfragen an Untersuchungen von Frauen in den 1940er und 50er Jahren anzuschließen und die neuen methodischen Ansätze zu integrieren. Seit der zweiten Hälfte der 1970er Jahre begann die Frauengeschichte auch an den deutschsprachigen Universitäten Fuß zu fassen. Für die europäische und die amerikanische Frauenforschung fungierte die Geschichtswissenschaft lange Zeit als Leitwissenschaft, und das hatte, wie Joan Scott feststellt, mit der Nützlichkeit historischer Argumentationen zu tun: „Feminism has used history in different ways and different times ... as a critical weapon in the struggle for women's emancipation. Feminist History has offered demonstrations, in the form of exemplary instances from the past, of women's worthiness to engage in the same activities as men ..." Nicht zuletzt habe feministische Geschichtsschreibung die Geschichten entlarvt, die beweisen wollten, dass die gesellschaftliche Rolle von Frauen naturgegeben sei. ${ }^{47}$

Joan Scott sagt, „feminist history was never primarily concerned with documenting the experiences of women in the past, even if that was the most visible means by which we pursued our objective. The point of looking to the past was to destabilize the present, to challenge patriarchal institutions and ways of thinking. "48 Und diese Intention kollidierte mit dem etablierten Geschichtsverständnis, selbst wenn es nicht

\footnotetext{
46 Brief an Bettina von Arnim, vom 6.2.1828, in: Leopold von Ranke, Das Briefwerk, hg. von Walter Fuchs, Hamburg 1949, 139, zit. lt. Smith, Gender, wie Anm. 29, 116.

47 Joan W. Scott, Feminism's History, in: Journal of Women's History, 16, 2 (2004), 10-29, 18 (dt.: Geschichte der Feministinnen, in: L'HOMME. Z. F. G., 14, 2 (2003), 317-336).

48 Scott, History, wie Anm. 47, 21.
} 
ausschließlich oder gar explizit darum ging, ,das Patriarchat' zu stürzen. Die politische Forderung nach Gleichstellung, die mit der Auseinandersetzung mit den ,Ungleichheiten' von Vergangenheit und Gegenwart und der Vorstellung verbunden war, dass diese Ungleichheiten nicht ,natürlich' waren, widersprach der Forderung, dass Geschichte um ihrer selbst willen studiert werden sollte. Zwar wissen wir, dass Ranke selbst durchaus nicht ohne politische Intentionen und schon gar nicht ohne politische Wirkung war. Seine historischen Interpretationen stützten den preußischen Staat mehr als jede Hofgeschichtsschreibung das hätte tun können. Es gelang ihm aber, nicht zuletzt mit dem Hinweis auf die ,unbestechlichen' Quellen, den Nimbus der Objektivität aufrecht zu erhalten.

Was die Frauengeschichte innerhalb der historischen community so verdächtig machte, ließ sie in der Frauenbewegung und in der in enger Beziehung dazu entstandenen interdisziplinären Forschung zum bedeutenden Faktor werden. Frauengeschichte war „part of a movement that consolidated the identity of women as political subjects, enabling activism in many spheres of society and winning unprecedented public visibility and, eventually, some success" ${ }^{49}$ wie Scott schreibt. Frauengeschichte war also äußerst nützlich. Sie war für die interdisziplinäre Frauenforschung und für die Forschung in anderen Fächern zentral. Historische Forschung konnte zeigen, wie und durch welche Vorstellungen, Konzepte, Institutionen, Gesetze oder Diskurse der Ausschluss von Frauen aus wichtigen Feldern etabliert und legitimiert wurde, beziehungsweise wie ihre Beschränkung auf andere Felder aufrecht erhalten werden konnte. Und das nahm der gesellschaftlichen Situation die Naturgegebenheit. Sie ließ zudem erahnen, wie unterschiedlich und wechselhaft die Vorstellungen von weiblichen Eigenschaften, die Forderungen, wie eine Frau zu sein hatte oder was das Wesen, die „Natur der Frau“ ausmachte, in der Geschichte waren. Erst das ermöglichte die Historisierung der Kategorie Frau.

Diese Entwicklung wurde durch die Einführung der Kategorie, Gender/Geschlecht akzentuiert. In der Mitte der 1980er Jahre führte die Gender-Theorie zur kritischen Beurteilung und Neuorientierung der Frauengeschichte. Der neue theoretische Ansatz postuliert ,Männlichkeit' und ,Weiblichkeit' als Set von in gegenseitiger Abhängigkeit geschaffener Charakteristika, die das Leben von Frauen und Männern prägen, ja konstruieren..$^{50}$ Dabei wird die Vorstellung zurückgewiesen, dass ,Männlichkeit und ,Weiblichkeit' oder ,Mann' und ,Frau' bestimmten biologischen Determinanten entspricht. ${ }^{51}$ Joan Scott zeigte in ihrem grundlegenden Aufsatz „Gender: A Useful Category

49 Scott, History, wie Anm. 47, 21.

50 Auch wenn sich Frauengeschichte nie ausschließlich mit Frauen beschäftigt hatte, sondern - entgegen anders lautender Kritik - die Geschlechterbeziehungen einbezogen hatte, erleichterte die Bezeichnung Geschlechtergeschichte oder Frauen- und Geschlechtergeschichte dies auch deutlich zu machen und forderte zudem dazu auf, auch Männer als Geschlechtswesen zu konzeptualisieren.

5I Bonnie G. Smith. Introduction, in: Womens' History in Global Perspective, Bd. 1, Urbana/Chicago $2004,2$. 
of Historical Analysis", 52 dass Gender selbst ein konstitutives Element im System der sozialen Beziehungen ist. Gesellschaftliche Symbole, normative Konzepte, politische und wirtschaftliche Prozesse, subjektive Identität beruhen auf Vorstellungen von Männlichkeit und Weiblichkeit - und reproduzieren sie.

Dass Sprache konstitutiv für Lebensverhältnisse ist und nicht nur reflexive Funktionen erfüllt, hat die Frauen- und Geschlechterforschung nicht erst geprägt, nachdem der linguistic turn Eingang in die Geschichtswissenschaft gefunden hatte. Historikerinnen, denen die ,biologischen Tatsachen' als Grundlage, Ausgangspunkt und Erklärung für die Ungleichheit zwischen den Geschlechtern zu kurz griffen, machten Macht und Wirksamkeit von Diskursen im sozialen Prozess der Konstruktion von Geschlecht und Geschlechterdifferenz und bei der Festschreibung von Ungleichheit in gesellschaftlichen Praktiken und Institutionen sichtbar. Feministische und poststrukturalistische Kritikerinnen hatten die Erklärungsmuster und Paradigmen der Geschichtswissenschaft hinterfragt, zerlegt und unter Einbezug der Bedeutung von Geschlecht, Rasse und Ethnizität neu geformt. ${ }^{53}$ Es hatte aber auch und gerade von Historikerinnen heftige Kritik gegenüber der Dominanz des linguistic turn und der Bedeutung der Diskurstheorie gegeben. Am Ausgangspunkt dieser Debatte zu Beginn der 1990er Jahre stand die antithetische Gegenüberstellung von Erfahrung und Diskurs und der Vorwurf von Joan Scott, die Geschlechtergeschichte habe den Begriff Erfahrung unkritisch verwendet und $\mathrm{zu}$ wenig berücksichtigt, dass Menschen Erfahrung aufgrund bereits bekannter Normen und Werte machen - und diese Werte auch diskursiv vermittelt werden. ${ }^{54}$ Dem wurde entgegengehalten, dass die Kategorie ,Erfahrung' infolge des linguistic turn ihre Bedeutung eingebüßt habe und dadurch das Erfahrung machende Subjekt in der Geschichtswissenschaft verloren gegangen sei. Inzwischen ist die Debatte nicht mehr durch den Vorwurf der Dominanz der Diskurse auf der einen oder der essentialistischen Interpretation von Erfahrung auf der anderen Seite geprägt, vielmehr steht die Frage nach der Beziehung von Diskurs und Erfahrung im Zentrum und hat auch die interdisziplinäre Geschlechterforschung befruchtet. So zielt die norwegischamerikanische Literaturwissenschaftlerin Toril Moi in ihrer Arbeit über Simone de Beauvoir darauf $a b$, ein historisiertes Verständnis von Körper und Subjektivität zu schaffen, bei dem der Körper die Grundlage, aber nicht die Determinante für Er-

52 Joan W. Scott, Gender: A Useful Category of Historical Analysis, in: American Historical Review, 91, 5 (1986), 1053-1075.

53 Vgl. Kathleen Canning, Feminist History after the Linguistic Turn. Historicizing Discourse and Experience, in: Signs. Journal of Women in Culture and Society, 19, 2 (1994), 368-404.

54 Vgl. Joan Scott, The Evidence of Experience, in: Citical Inquiry, 17, 3 (1991), 773-797; auch: Marguérite Boz, Bettina Vincenz u. Tanja Wirz, Erfahrung: Alles nur Diskurs? Auseinandersetzung mit einer Debatte um einen vielschichtigen Begriff, in: dies. Hg, Erfahrung: Alles nur Diskurs? Zur Verwendung des Erfahrungsbegriffes in der Geschlechtergeschichte. Beiträge zur 11. Schweizerischen HistorikerInnentagung, Zürich 2004, 9-21. 
fahrungen abgibt. ${ }^{55}$ Auch in den Debatten um den Körper, die Judith Butler in „Bodies that matter" führt, ist die historische Dimension, wenn auch implizit, ebenso vorhanden, wie in dem Konzept von ,Geschlecht als Existenzweise' der Philosophin und Soziologin Andrea Maihofer. ${ }^{56}$

Die Frauen- und Geschlechtergeschichte verdankt den interdisziplinären, dekonstruktivistischen Ansätzen der Geschlechterforschung wichtige Erkenntnisse über die Konstruktionsmechanismen von Männlichkeit und Weiblichkeit und die Brüchigkeit der Kategorie ,Frau' (und ,Mann'). ${ }^{57}$ Geschlecht/Gender hat sich also ohne Zweifel als nützliche Kategorie für die historische Forschung erwiesen. Dennoch wurde gerade in letzter Zeit erneut ${ }^{58}$ ein Unbehagen darüber artikuliert, dass in der postmodernen Theorie der feministische Anspruch, Frauen ins Zentrum der Überlegungen zu stellen, als naiv und altmodisch abgetan wird, und es kaum noch gelingt das Wort ,Frau' auszusprechen, ohne damit schwere metaphysische Implikationen zu verbinden, wie Toril Moy ironisch bemerkt hat. ${ }^{59}$

Judith Bennett kritisiert nicht nur - wie bereits eingangs erwähnt - die fehlende praktische (und politische?) Resonanz der theoretischen Ansätze. Sie führt weiterhin aus, warum sie - trotz hoher Wertschätzung der theoretischen Konzeptionen der Geschlechterforschung - fortfährt, von ,Frauen' statt von ,Gender' zu sprechen und warum ihr das Aufgehen der Frauengeschichte in der Geschlechtergeschichte problematisch erscheint: ,Frau' sei zwar theoretisch eine flüchtige („slippery“) Kategorie, in der Praxis aber - zu einer bestimmten historischen Zeit und an einem bestimmten Ort - fungiere sie als feste („stable“) Kategorie, die geeignet sei, die Lebensperspektiven einer Person zu bestimmen. Dabei ist ihr das „caveat“ äußerst wichtig, dass es sich um Stabilität „for it’s time and place" handle. ${ }^{60}$

55 Vgl. Toril Moi, What is a Woman? And Other Essays, New York 1999; dies., Simone de Beauvoir. Die Psychographie einer Intellektuellen, Frankfurt a. M. 1996; vgl. Kathleen Canning, Problematische Dichotomien. Erfahrungen zwischen Narrativität und Materialität, in: Historische Anthropologie, 10, 2 (2002), 163-182 (Nachdruck in Marguérite Bos, Bettina Vincenz u. Tanja Wirz Hg., Erfahrung: Alles nur Diskurs? Zur Verwendung des Erfahrungsbegriffs in der Geschlechtergeschichte, Zürich 2004).

56 Vgl. Judith Butler, Bodies that Matter. On the Discursive Limits of „Sex“, New York/London 1993; Andrea Maihofer, Geschlecht als Existenzweise. Macht, Moral, Recht und Geschlechterdifferenz, Frankfurt a. M. 1995.

57 Bennett, History, wie Anm. 3, 9.

58 In den Anfängen der Auseinandersetzung mit der Konzeption von Geschlecht/Gender als Kategorie war bekanntlich befürchtet worden, dass die neue Begrifflichkeit Frauen wiederum unsichtbar mache und so die politischen Forderungen nach Emanzipation von Frauen schwächen würde. Weiterhin hatten gerade auch Historikerinnen betont, dass der immer noch bestehende eklatante Nachholbedarf an Wissen über Frauen, weiterhin Frauengeschichte unter dieser Begrifflichkeit legitimieren würde; vgl. Regina Wecker, Zwischen Ökonomie und Ideologie. Arbeit im Lebenszusammenhang von Frauen im Kanton Basel-Stadt 1870-1910, Zürich 1997, 9ff.

59 Moi, Woman, wie Anm. 55, 10. 
In der historischen Forschung muss es also immer auch darum gehen, diese ,feste Kategorie zu erfassen, das, was für die ZeitgenossInnen das, Wesen einer Frau' ausmacht. Mir ist in diesem Zusammenhang noch eine weitere Überlegung wichtig: Historische Forschung muss nicht nur zeigen, was die für einen bestimmten Zeitraum und für einen bestimmten Kontext feststehenden Geschlechterkonzeptionen sind, wie und innerhalb welcher Grenzen sie bezüglich Schicht, Klasse, Rasse oder Ethnie variieren, sondern sie muss auch zeigen, welche Merkmale der Kategorie sich über sehr lange Zeiträume als stabil erweisen. Die ,Amateurin', wie sie etwa durch die Professionalisierung der Geschichtswissenschaft entstanden ist, erscheint mir als treffendes Beispiel dieser Stabilität, das uns seit dem 19. Jahrhundert in vielfältigen Zusammenhängen, Berufsfeldern und Kontexten begegnet.

Diese Stabilität von Merkmalen kann den Aussagen der zeitgenössischen Quellen widersprechen, insbesondere dann, wenn es um die Legitimationen von neuen wissenschaftlichen ,Erkenntnissen' geht, und gerade das Neue zum zentralen Qualitätskriterium gemacht wird. So wird der Schweizer Psychiater und Wegbereiter der Eugenik, August Forel, unter Hinweis auf die wissenschaftlichen Erkenntnisse der neuen Vererbungsforschung die politischen Gleichberechtigung von Frauen fordern, da die männlichen und die weiblichen Gene sozusagen ,gleichberechtigt' sind, aber gleichzeitig die Merkmale der ,alten' Geschlechtscharaktere beibehalten, wie sie im 18. und 19. Jahrhundert geformt wurden. ${ }^{61}$

Die Herausforderung für die HistorikerInnen besteht darin, diese Stabilitäten - die kontextgebundenen, wie die langfristigen, epochenübergreifenden - zu analysieren, ohne dabei die Flüchtigkeit und Brüchigkeit zu negieren oder den Eindruck zu erwecken, dass sie selbst von einem Verständnis der Kategorie ,Frau' ausgehen, das alle anderen Kategorien transzendiert. Dennoch ist für die Gender-Theorie, die sehr einheitlich auf die Brüchigkeit und Flüchtigkeit von Kategorien setzt, diese doppelte Ausrichtung der historischen Forschung zumindest immer wieder erklärungsbedürftig. Wenn zudem die Nützlichkeit der historischen Forschung gerade auch darin bestanden hatte, zu zeigen, wie sich die Konzepte veränderten, weil gerade das es ermöglicht hatte, den Geschlechterverhältnissen den Anstrich der Natürlichkeit zu nehmen, dann ist ein möglicher Grund für den anfänglich angesprochenen Bedeutungsverlust der Geschichte innerhalb der Gender Studies, dass diese Festigkeit naiv oder gar bedrohlich wirkt.

6I Etwa in Form der Gegensatzpaare aktiv - passiv, listig - brutal, monogam - poligam, leidenschaftlich unschöpferisch. Vgl. Auguste Forel, Die sexuelle Frage. Eine naturwissenschaftliche, psychologische, hygienische und soziologische Studie für Gebildete, München 1905. 


\section{Der Erfolg der Frauen- und Geschlechtergeschichte und der Verlust des Fortschritts-Paradigmas}

Mit dem folgenden Abschnitt möchte ich die Entwicklung der Frauen- und Geschlechtergeschichte nochmals aus einer anderen Perspektive angehen und dabei auf die Veränderungen der Beziehung zur sogenannt allgemeinen Geschichte und zur Wissenschaftsgeschichte fokussieren.

In den Anfängen trat Frauengeschichte an Tagungen, in Lehrveranstaltungen und Publikationen meist in einem interdisziplinären Kontext auf. Disziplinäre Grenzen schienen wenig wichtig, auch das machte übrigens den Ansatz innerhalb des historischen Fachs verdächtig. Der Erfolg der Frauen- und Geschlechtergeschichte führte zu einer gewissen Institutionalisierung und vice versa führte die Institutionalisierung auch zu weiteren Erfolgen. Das veränderte die historische Disziplin, veränderte aber auch die Frauen- und Geschlechtergeschichte.

Geschlechtergeschichte ist nicht zum Mainstream innerhalb der Geschichtswissenschaft geworden. „We have never been really accepted“, sagt selbst Joan Scott. Aber die Geschlechtergeschichte entwickelte engere Verbindungen zur historischen Disziplin, zu deren Methoden, Konzepten und Potentialen.

Engere Beziehungen zur Geschichtswissenschaft zu haben, heißt keineswegs, die anderen Disziplinen zu ignorieren. ${ }^{62}$ Aber die Frauen- und Geschlechtergeschichte fragte zunehmend stärker nach Erfahrungen von Frauen und Männern in der Vergangenheit als nach emanzipativen Tendenzen und Wirkungen. Die Unterschiede der Epochen und deren spezifische Entwicklungen wurden wichtiger als die Frage, welche Bedeutung sie für gegenwärtige Entwicklungen hatten. Man könnte also sagen, dass Epochen, Ereignisse und Entwicklungen wieder Bedeutung ,um ihrer selbst willen' erhielten. Es wurde weniger wichtig zu zeigen, wie und warum Frauen diskriminiert wurden, und was das für die heutige Situation bedeutete. Selbst die Darstellung weiblicher (Mit-)Täterschaft hatte den revolutionären Impetus verloren. Michelle Perrot hatte das bereits 1989 angesprochen, als sie ausführte, dass es wichtiger sei, die „sozialen Praktiken, die verschiedenen Diskurse, die eigentümlichen Repräsentationen und Vorstellungen zu untersuchen und sich freizumachen von grobschlächtigen Dichotomien wie ,Natur/Kultur' oder ,häuslich/ öffentlich'; damit aufzuhören, die ,Misogynie‘ als stets passende Erklärung für den Ort der Frauen in der Gesellschaft zu gebrauchen; sich endlich mit den Grauzonen, den Widersprüchen ... zu beschäftigen, die Ambivalenzen ernster zu nehmen als die grellen Plakatfarben ..." ${ }^{“ 3}$ Eine solche differenzierte, nicht homogenisierende, nicht plakative

62 Susanna Burghartz, Umordnung statt Unordnung? Ehe, Geschlecht und Reformationsgeschichte, in: Helmut Puff u. Christopher Wild Hg., Zwischen den Disziplinen? Perspektiven der Fühneuzeitforschung, Göttingen 2003, 165-185.

63 Michelle Perrot 1989 zit. lt. Claudia Opitz, Um-Ordnungen der Geschlechter. Einführung in die 
Geschichtsschreibung ist allerdings weniger nützlich für politische Forderungen, sie lässt sich weniger schnell als Politikberatung einsetzen.

Dass man sich auch und gerade auf Widersprüche und Grauzonen einließ, brachte eine weitere Veränderung mit sich: Joan Scott hat das „the loss of the grand teleological narrative of emancipation“ genannt, den Verlust der Selbstverortung „on the side of redemptive history“. ${ }^{64}$ Diese Vorstellung lässt sich auf weitere Felder als die Fragen des Emanzipationsfortschritts im politischen, gesellschaftlichen und rechtlichen Bereich beziehen: nämlich auf die Geschichtsschreibung selbst. Damit spreche ich Fragen an, die auch für das Wissenschaftsverständnis der Frauen- und Geschlechtergeschichte zentral sind, Fragen, die die Historizität von Wahrheit und Wissenschaft aber auch und insbesondere das Verhältnis von Kontinuitäten und Brüchen betreffen. Die Konzepte und Darstellungen zweier Wissenschaftler sind in diesem Zusammenhang von besonderer Bedeutung: von Ludwik Fleck und von Michel Foucault.

Ob etwas im wissenschaftlichen Sinne als wahr gilt, hängt davon ab, ob es unter den gegebenen historischen Umständen und mit den (zeit-)spezifischen Beweis-Methoden Plausibilität erlangen kann. Fleck hat die Grundbedingungen für diesen Plausibilitätsnachweis eindrücklich beschrieben und insbesondere die historische Bedingtheit auch der naturwissenschaftlichen „Tatsachen“ betont, wenn er ausführt, dass es in „den Naturwissenschaften ... gleichwie in der Kunst und im Leben keine Naturtreue als die Kulturtreue" gäbe. ${ }^{65}$ Dabei geht es ihm um die Kontinuität von wissenschaftlichem und außerwissenschaftlichem Wissen und um die Kontextgebundenheit des Wissens, und auch darum, wie überkommene, traditionelle Vorstellungen das Verständnis und das ,Sehen' des Wissenschaftlers prägen. Er relativiert aber nicht nur die Vorstellung von eindeutigen naturwissenschaftlichen, Wahrheiten'. Er verschafft - darauf kommt es mir in diesem Kontext besonders an - der Geschichte und der Geschichtswissenschaft eine wichtige Legitimation: Erst die Beschäftigung mit vergangenen Wissenssystemen erlaube es, gegenwärtiges Wissen umfassend - wenn auch nicht endgültig - deuten zu können. Stärker lässt sich die Bedeutung einer langfristigen historischen Perspektive kaum formulieren. Michel Foucault dagegen fokussiert auf ,Brüche‘, ,Diskontinuitäten ‘ und,Widersprüche“ in der Geschichte des Bewusstseins. Beide gehen von der Kontextabhängigkeit wissenschaftlicher Erkenntnisse aus und sind an deren kultureller Bedingtheit interessiert. Foucault geht es explizit nicht um die inhaltliche Weiterentwicklung wissenschaftlicher Erkenntnisse, nicht um den Zugewinn an Erkenntnissen innerhalb einer historischen Epoche, sondern um die Regeln, Bedingungen und Modalitäten der Ordnungen, denen sie unterworfen waren. ${ }^{66}$ Es ist also zu fragen, was es für die His-

\footnotetext{
64 Scott, History, wie Anm. 47, 13.

65 Ludwik Fleck, Entstehung und Entwicklung einer wissenschaftlichen Tatsache. Einführung in die Lehre vom Denkstil und Denkkollektiv, Frankfurt a. M. 1980, 48 (Orig. 1935).

66 Michel Foucault, Die Ordnung der Dinge. Eine Archäologie der Humanwissenschaften, Vorwort zur deutschen Ausgabe, Frankfurt a. M. 1989, 15 u. 24.
} 
toriographiegeschichte und für die Geschlechterforschung bedeuten würde, wenn auch Geschichtsschreibung nicht unter der Vorstellung des Zugewinns an Erkenntnis betrachtet würde, nicht als Vorgeschichte heutigen wissenschaftlichen Denkens, sondern wenn stattdessen ihre Produktionsbedingungen und die Frage, „wie sie funktioniert“, im Zentrum stehen würden und wenn wir zudem die Frage nach den Machtverhältnissen, wie sie Foucault stellt, um die Frage der darin enthaltenen Geschlechterverhältnisse ergänzen. ${ }^{67}$ Ich möchte daran mit der Geschichte der Eugenik anschließen.

\section{Eugenik als Wissenschaft und Text}

Eugenik und Vererbungsforschung waren die neuen Wissenschaften des 20. Jahrhunderts, die die Darwin'schen wissenschaftlichen Erkenntnisse der Evolutionstheorie aufnahmen. Die Geschichte der Eugenik in der Schweiz erscheint mir geeignet, einige der am Beispiel der Frauen- und Geschlechtergeschichte dargestellten Wertungen zu verdeutlichen und auf sozialgeschichtlich orientierte Forschung anzuwenden. Dabei werde ich methodisch die Forderung von Helmut Puff aufnehmen, Wissenschaftsgeschichte nicht nur als Ideengeschichte, sondern auch als Textgeschichte zu begreifen. Dies scheint mir für die Geschichte der Eugenik, die Elemente der Naturwissenschaften, der Sozialwissenschaften und ihrer Umsetzung in politische Maßnahmen vereint, besonders nützlich. An die Ausführungen zur Frauen- und Geschlechtergeschichte schließe ich dabei insbesondere mit Fragen nach Bruch und Kontinuität, nach der Bedeutung der Langfristigkeit von Entwicklungen und nach Charakteristika und Transformationen der Moderne an.

Wie in anderen europäischen Ländern und in den USA waren eugenische wissenschaftliche Konzepte auch in der Schweiz zu Beginn des 20. Jahrhunderts auf geradezu flächendeckendes Interesse gestoßen. ${ }^{68}$ In der Eugenik flossen neue wissenschaftliche Erkenntnisse zusammen, wie die Übertragung der Mendel'schen Vererbungslehre auf den Menschen, psychiatrische Vorstellungen über die Vererbbarkeit von psychischen Defekten und erste Erkenntnisse über genetische Erbinformationen. Dass im nationalsozialistischen Deutschland eugenische Konzepte zu zwangsmäßigen Massensterilisierungen und schließlich zu Massenmorden in psychiatrischen Kliniken geführt hatten, verdeckte lange, dass die Vorstellungen, mit eugenischen Maßnahmen den „Volkskörper" zu verbessern, auch in nicht autoritären Regimes Verbreitung gefunden hatten. Die in der Schweiz angewandten Maßnahmen zur Verhinderung „erbkranken Nachwuchses" reichten von (Ehe-)Beratung und Heiratsverboten für Schwachsinnige, wie es im Zivilgesetzbuch (ZGB) 1912 verankert wurde, bis zu eugenischen Bestimmungen in

67 Epple, Historiographiegeschichte, wie Anm. 31, 80ff.

68 Vgl. Regina Wecker, Eugenik. Individueller Ausschluss und nationaler Konsens, in: Sebastien Guex 
Einbürgerungsgesetzen und zu Sterilisationen. Sterilisation stellen im Schweizer Kontext die radikalste Maßnahme der Grenzziehung zwischen sogenannt „vererbungswürdigen" und „vererbungsunwürdigen" Anlagen dar. ${ }^{69}$

Es war Konsens unter den Experten, dass in der Schweiz Sterilisationen nur akzeptiert würden, wenn sie freiwillig erfolgten. Überwiegend waren die Sterilisierten anders als im nationalsozialistischen Deutschland - Frauen. ${ }^{70}$ Die Betroffenen mussten schriftlich in die Maßnahmen einwilligen, allerdings gab es vielfältige Möglichkeiten, diese Einwilligung zu erzwingen, etwa indem die Entlassung aus einer Anstalt davon abhängig gemacht, oder die Sterilisation zur Voraussetzung für finanzielle Unterstützung deklariert wurde. Diese Situation lässt nach der Bedeutung und Definition von Freiwilligkeit fragen. Wie ,gendered' war das Konzept der Freiwilligkeit? Die Interpretation liegt nahe, dass Frauen auch in dieser Frage an der aufgeklärten Vorstellung vom freien Willen als unverzichtbarem Bestandteil des, modernen' Menschen nicht teilhatten.

Wie die Sterilisation war auch die Abtreibung in der Schweiz in den 1930er Jahren nur aus medizinischen Gründen gesetzlich erlaubt, also wenn ein Gesundheitsrisiko für die Frau bestand. Aber schon in den 1930er und 40er Jahren konnten Frauen abtreiben lassen, wenn sie selbst oder aber der Arzt eugenische Gründe und soziale Not geltend machten, das heißt Krankheiten, wie Schwachsinn, Geisteskrankheit, Schizophrenie, Epilepsie oder ähnliche anführten, deren Definition eine Amalgamierung von medizinischen, eugenischen und sozialen Gründen möglich machte. Zudem war es in diesem Kontext ,hilfreich', dass die Unterscheidungen ,ordentlich - unordentlich', , normal anomal' sehr deutlich entlang normativer Vorstellungen von Geschlecht verliefen. Nun war eine „Erbkrankheit" keine temporäre Angelegenheit, sodass die Frage, ob eine Abtreibung eine Sterilisation nach sich ziehen sollte, oft latent mitschwang. Die Situation schuf also Möglichkeiten, Druck auf Frauen auszuüben: Für eine - auch von der Frau - gewünschte Abtreibung konnte eine allenfalls nicht erwünschte Sterilisation zur Bedingung gemacht werden. Dies erklärt zumindest teilweise, warum - entgegen dem eugenischen Wissen, dass die Erbanlagen von Männern und Frauen bei der Entstehung von Erbkrankheiten gleich wichtig sind - mehrheitlich Frauen sterilisiert wurden. Als weiterer Grund für dieses Geschlechterverhältnis war die schlechtere soziale

69 Vgl. die Ergebnisse des Forschungsprojekts „Eugenische Konzepte und Massnahmen in Psychiatrie und Verwaltung. Zur Politik von Normierung, Integration und Ausgrenzung am Beispiel des Kantons Basel-Stadt, 1880-1960“ (Ltg. Regina Wecker u. Bernhard Küchenhoff); vgl. auch die Projekte „Städtische Fürsorge im Kräftefeld von Eugenik, Geschlecht und medizinisch-psychiatrischen Normalisierungsdiskursen in Bern und St. Gallen (1918-1955)“ (Ltg. Béatrice Ziegler u. Gisela Hauss) u. „Internieren und Integrieren. Zwang in der Psychiatrie: Der Fall Zürich, 1870-1970“ (Ltg. Jakob Tanner). Die Projekte sind im Rahmen des „Nationalen Forschungsprogramms 51 - Integration und Ausschluss" durchgeführt worden.

70 Vgl. Gisela Bock, Zwangssterilisation im Nationalsozialismus. Studien zur Rassenpolitik und Frauenpolitik, Opladen 1986, 8. 
Stellung der Frauen, die Tatsache, dass sie eher gezwungen waren, dem Druck nachzugeben, aber auch dass die männlichen Ärzte sehr viel zurückhaltender waren, anderen Männern einen Eingriff, vorzuschlagen', der so tief in das männliche Selbstverständnis von physischer und psychischer Potenz eingriff.

Die Frage von Verhütung und Abtreibung ist eine zentrale Frage der feministischen Politik. ${ }^{71}$ Das Recht auf Abtreibung war zum Beispiel in der deutschen Frauenbewegung der 1970er Jahre ( $\$ 174)$ ein Brennpunkt feministischer Politik. Die voraussetzungsfreie Abtreibung und die Abschaffung des psychiatrischen Gutachtens, wie sie das neue Schweizer Gesetz zum Schwangerschaftsabbruch 2002 garantiert, wurden wie das Recht über die Zahl der Kinder zu bestimmen - als Sieg der Frauenbewegung gewertet.

Sowohl bei der Abtreibung als auch bei der Sterilisation ging es darum, eine moderne ,Technik' zu nutzen, die durch die Eugenik Vervollkommnung, Verbreitung und wohl auch gesellschaftliche Akzeptanz gefunden hatte. Schließlich ermöglichte sie die Verhinderung unerwünschter und, nach der Vervollkommnung medizinischer Kontrollmöglichkeiten, auch kranker und behinderter Nachkommen. Hatte die Eugenik damit sozusagen „enabling forces“, wie die dänische Historikerin Lene Koch ${ }^{72}$ in einem sehr interessanten und subtilen Artikel meinte? Koch verwirft - unter Hinweis auf das Foucault'sche Konzept der Biopolitik - die Grenzziehung, die moderne Genetiker zwischen ihrer Wissenschaft und der Eugenik vorgenommen haben. Der Genetik war es nach 1945 gelungen, sich als neue Wissenschaft zu etablieren, weil sie die neuen Erkenntnisse als wissenschaftlich legitimiert bezeichnete, während die Eugenik als Pseudowissenschaft abqualifiziert wurde. Die Genetik machte diesen Bruch mit der Behauptung glaubhaft, dass sie auf den erweiterten Handlungsspielraum des Individuums gerichtet sei, während die Eugenik den „Volkskörper“ über das Individuum gestellt und dessen Handlungsspielraum eingeschränkt habe. Die Geschichtsschreibung hatte lange Zeit sowohl diese Vorstellung eines Bruches anerkannt als auch die Konzeption von Eugenik als nationalsozialistischer Wissenschaft fortgeschrieben. ${ }^{73}$

Wurde Eugenik zu Unrecht als antifeministisch, konservativ, unwissenschaftlich bezeichnet? Bereitete die Eugenik den Weg zur sexuellen Selbstbestimmung vor?

7I Linda Gordon, Woman's Body, Woman's Right. A Social History of Birth Control in America, New York 1976.

72 Lene Koch, How Eugenic was Eugenics? Reproductive Politics in the Past an the Present, in: Regina Wecker, Sabine Braunschweig, Gabriela Imboden, Bernhard Küchenhoff u. Hans Jakob Ritter Hg., Wie nationalsozialistisch ist die Eugenik? What is National Socialist about Eugenics? Beitrag zur Geschichte der Eugenik im 20. Jahrhundert. Contribution to the History of Eugenics in the 20th Century (ersch. Ende 2007); vgl. Lene Koch, The Meaning of Eugenics. Reflections on the Government of Genetic Knowledge in the Past and the Present, in: Science in Context, 17, 3 (2004), 1-17.

73 Vgl. Peter Weingart u. a., Rasse Blut und Gene. Geschichte der Eugenik und Rassenhygiene in Deutschland, Frankfurt a. M. 1992. 
Schließlich hatte auch die Schweizer Frauenbewegung das Werk des einflussreichen Psychiaters und Sexualwissenschaftlers Auguste Forel „Die sexuelle Frage" ${ }^{\text {"74 }}$ mit großem Interesse aufgenommen, weil er Sexualität und Fortpflanzung trennte, Frauen eine eigene Sexualität zugestand und für ihre politische Emanzipation eintrat. Auch hatten die Eugeniker selbst betont, dass die Eugenik das progressive, humanitäre rationale Projekt sei, das Menschen re-integriere, statt sie, wie früher, einfach einzusperren und so aus der Gesellschaft auszuschließen.

Für Zygmunt Bauman hingegen war die Eugenik, die Kontrolle des Erbgutes und die eugenische Selektion „einfach ein radikaler Ausdruck der allgemeinen Ambitionen, die der modernen Mentalität inhärent waren " ${ }^{75}$ Er sieht sie als Ausdruck des unbedingten Ordnungswillen und der Intention der Moderne, den Zufall auszuschalten und die Natur zu beherrschen. ${ }^{76}$ Dazu aber mussten die bisher unkontrollierten Kräfte der Vererbung eliminiert werden. Rassekonzepte erwiesen sich als geeignet, diese Notwendige Unterscheidung zwischen erwünschten und unerwünschten Individuen zu markieren, auch in der Schweiz. Auguste Forel, Lehrer und Mentor vieler deutscher Eugeniker, ist ein besonders erschreckendes Beispiel. Seine Schriften sind durchsetzt von rassistischen Konzeptionen. Er spricht von „minderwertigen Rassen“, insbesondere „Neger" werden als „geistig minderwertig“ bezeichnet, als „,impulsiv und willenschwach“, aber als sexuell besonders aktiv. ${ }^{77}$ Die „gelbe Rasse“ rechnet er gnädig zur „Kulturmenschheit", aber auch ihre große Fruchtbarkeit sei beunruhigend. Auch wenn er die europäischen „Rassen“ als gleichwertig einschätzt, sei innerhalb ihrer Gesellschaften zwischen „wertvollen“ und „minderwertigen“ zu unterscheiden. Letztere, die „Schmarotzer“, „Schädlinge“, diejenigen, die „vererbbare Krankheiten“ haben oder „krankhafte Konstitutionen“, „zu Tuberkulose Neigende“, „Alkoholsüchtige“, „Verbrecher“ und „Verbildete“ sollten von der Fortpflanzung ausgeschlossen werden. ${ }^{78}$ Allerdings spricht Forel den „Minderwertigen“ nicht das Recht auf Leben, sondern ,nur' das Recht auf Fortpflanzung ab - hier unterscheidet er sich von der späteren nationalsozialistischen Umsetzung eugenischer Vorstellungen. Verbal wird allerdings die Unterscheidung zwischen „lebenswert“ und „lebensunwert“ getroffen, auch wenn die vorgeschlagenen Maßnahmen das Recht auf Leben nicht absprechen. Insgesamt zielt er darauf ab, den physischen Ausschluss der ,anderen 'vom eigenen Volkskörper zu legitimieren. ${ }^{79}$

74 Forel, Frage, wie Anm. 61.

75 Bauman, Moderne, wie Anm. 38, 60.

76 Bauman, Moderne, wie Anm. 38, 61.

77 Zitate aus: Forel, Frage, wie Anm. 61; ders., Malthusianismus oder Eugenik?, München 1911 u. ders., Zur Rassenfrage in den Vereinigten Staaten, in: Neue Zürcher Zeitung, 19.7.1910.

78 Forel, Frage, wie Anm. 61, 253.

79 Vgl. Mirjam Bugmann u. Philipp Sarasin, Forel mit Foucault. Rassismus als ,Zäsur` im Diskurs von August Forel, in: Studien und Quellen. Zeitschrift des Schweizerischen Bundesarchivs, 29 (2003), 43-69. 
Deutlich wird, dass sich zwei Charakteristika der Moderne in der Schweiz im Widerstreit befanden: der Wunsch nach Ordnung und die Akzeptanz eines individuellen Selbstbestimmungsrechts, das das Schweizer Rechtssystem seit der Mitte des 19. Jahrhunderts garantiert. Das Selbstbestimmungsrecht von Frauen und von einigen wenigen Männern, die sich durch ihr Verhalten außerhalb der sexuellen Ordnung gestellt hatten, ${ }^{80}$ wurde als zweitrangig bewertet. Hier werden nun Kontinuitäten zu älteren Rechtsvorstellungen erkennbar, die zunächst durch die neuen Methoden der eugenischen Wissenschaft und durch den Wunsch der Wissenschaftler (und der wenigen Wissenschaftlerinnen) das Neue zu verkörpern und mit ihren Theorien die Utopie einer neuen Gesellschaft ohne „Minderwertige“ und Kranke zu verwirklichen, verdeckt wurden.

Bis zum 20. Jahrhundert war die Kontrolle der weiblichen Sexualität eng mit moralischen Kategorien verknüpft. Außereheliche Sexualität wurde bis weit ins 19. Jahrhundert bestraft. Sexualität galt nicht als Privatsache. Auch die Eheschließung war keine Angelegenheit zwischen nur zwei Personen. Die Gemeinden wollten auch im 19. Jahrhundert die traditionelle Kontrolle über Eheschließungen ausüben, zumindest über die ärmeren Bürger: Sie verlangten den Nachweis ausreichender finanzieller Mittel. Dabei ging es insbesondere darum, zu verhindern, dass der Gemeinde durch Kinder minderbemittelten Paare Kosten entstanden. Es wirkten aber wohl auch noch ältere Vorstellungen, die die Eheschließung durchaus nicht als ein individuelles Recht sahen und eine Kontrolle der sozialen Verhältnisse anstrebten. Diese Kontrollmöglichkeit wird den Gemeinden in der Schweiz erst in der Bundesverfassung von 1874 genommen, die ökonomisch motivierte Eheeinschränkungen verbietet.

Das moderne Rechtssystem gestand nun der Gesellschaft die moralische Kontrolle, die früher über Heiratsregeln, später über Heiratsverbote oder Strafen bei unehelicher Schwangerschaft ausgeübt werden konnte, nicht mehr zu. Aber für bestimmte Bevölkerungsgruppen - soziale Außenseiter, insbesondere der Unterschicht - wurde, über den Weg der eugenischen Argumentation, die all das zum Wohle des „Volkskörpers“ legitimierte, ein Weg eröffnet, diese Kontrolle wieder einzuführen: Das ZGB von 1912 verbot „Geisteskranken“ - die Definition war äußerst schwammig - die Eheschließung. Die Auslegung der betreffenden Bestimmung eröffnete zudem die Möglichkeit, die Eheschließung von einer Sterilisation abhängig zu machen.

Die neue Wissenschaft Eugenik und ihre statistischen ,Wahrheitsbeweise', die neuen Techniken der Sterilisation und der Abtreibung ermöglichten auf spezifische Weise die Fortführung traditioneller Kontrollen. Für die Historiographiegeschichte der Eugenik kommt dabei ein weiterer Faktor hinzu, der bisher erstaunlicherweise kaum ausreichend berücksichtigt wurde: Ein wesentlicher Teil der Akzeptanz eugenischer Paradigma in Psychiatrie und Genetik in den nicht-totalitären Gesellschaften, aber auch in Deutschland vor der Zeit des Nationalsozialismus, dürfte gerade darauf zurückzuführen

8o Homosexuelle, Sexualstraftäter. 
sein, dass die Beurteilungen - und schließlich noch deutlicher die Maßnahmen - Frauen betrafen, trotz der geschlechtsneutralen Formulierungen.

Wenn man eugenische Sterilisationen als Fortsetzung von traditionellen Maßnahmen der sozialen Kontrolle, insbesondere der Frauen ärmerer Schichten sieht, wird einerseits deutlich, warum die Eugenik auf so fruchtbaren Boden fiel: Sie konnte sich neu, modern und wissenschaftlich gebärden, den Bruch mit der Vergangenheit vollziehen und doch an nur allzu Bekanntes anschließen. Andererseits erscheint bei Einbezug dieser Kontinuitäten die Frage nach dem Anteil der Eugenik am Prozess der Vorbereitung der sexuellen Selbstbestimmung in anderem Licht. Wenn wir fragen, „wer, warum und zu wessen Gunsten mit welchen Ergebnissen handelt“, so müssen wir zunächst festhalten, dass die eugenischen Maßnahmen ein sehr ähnliches Spektrum von Frauen treffen, wie die alten Strafbestimmungen, nämlich die, die sich nicht an die Geschlechternormen hielten, diejenigen, die nun in den programmatischen Schriften der Eugeniker als „Schwachsinnige“, „moralisch Verdorbene“, „geistig Zurückgebliebene“, „moralisch Schwachsinnige“ bezeichnet wurden. Von deren Nachkommen soll „der Volkskörper“ frei gehalten werden. Neben diesen neuen eugenischen Bezeichnungen, kommt selbst das alte Wort „Unzucht“ wieder vor, das noch stärker auf vormoderne Traditionen verweist. ${ }^{81}$

In den Krankenakten, ${ }^{82}$ die für alle Patienten und Patientinnen angelegt wurden, wird diese Tradition armenpolizeilicher Maßnahmen seit dem Beginn des 20. Jahrhunderts und bis weit in die Zeit nach dem Zweiten Weltkrieg noch viel deutlicher sichtbar. Anders als in den programmatischen Schriften eines Forel, wird hier erstaunlicherweise kaum noch vom „Volkskörper“ gesprochen. Eugenische Argumente wie die Verbesserung des Erbgutes und die Erb-Selektion werden nicht angesprochen, die Erbkrankheit des zu erwartenden Nachwuchses erscheint als nebensächlich. Im Vordergrund stehen die häuslichen Fähigkeiten der Frau und die ökonomische Frage, ob sie mit ihrem Kind der Gemeinde zur Last fallen wird. Hier wird verstärkt Kontinuität sichtbar. Das Neue, das die zeitgenössischen Eugeniker betonen, die Bedeutung der neuen Wissenschaft, tritt in den Krankenakten in den Hintergrund.

Allerdings gab es auch schon in den 1930er und 40er Jahren Frauen, die dem Sterilisationsdruck widerstanden und trotzdem eine Abtreibung durchsetzen konnten. Sie nutzten das eugenische Umfeld für den Ausbau der individuellen Entscheidungskompetenz, und leiteten eine Entwicklung ein, die es schließlich als individuelle Entscheidung einer Frau erscheinen ließ, ob und wie viele Kinder sie haben wollte. Noch die Selbstbezichtigung französischer Feministinnen und Abtreibungsbefürworterinnen,

8I Susanna Burghartz, Unzucht, in: Historisches Lexikon der Schweiz (HLS), unter <www.hls-dhsdss.ch>, Zugriff 24.8.2007.

82 Im Rahmen des NFP51-Projekts wurden von Sabine Braunschweig, Gabriela Imboden u. Hans Jakob Ritter ca. 800 Krankendossiers der Psychiatrischen Universitätsklinik Basel und ca. 490 Dossiers der Psychiatrischen Poliklinik Basel aus dem Zeitraum von 1880-1960 ausgewertet. 
die deutsche Kampagne zur Abschaffung des $₫ 218$ in den 1970er Jahren und die späteren Kampagnen der Schweizer Frauenbewegung zeigen allerdings, dass es vor allem gut informierte und gebildete Frauen waren, die die damals noch illegalen Möglichkeiten der Abtreibung genutzt hatten. ${ }^{83}$

Diese Langzeitperspektive eröffnet eine neue Sichtweise auf die unmittelbare Vergangenheit. Sie erlaubt hier wieder deutlich an die These von Ludwik Fleck anzuschließen, wonach die Vergangenheit immer in den Argumenten und Lösungsmustern der Gegenwart eingeschlossen sei. Sie zeigt aber auch, wie wichtig der Einbezug einer länger zurück liegenden Entwicklung ist, für den Judith Bennet für die Frauengeschichte so eindrücklich plädiert. Gerade die Langfristigkeit einer Entwicklung und ihre Ambivalenz lässt auf die weitere Fortführung schließen: Es werden zur Verhinderung von ,Fehlern' und ,Defekten' intensive vorgeburtliche Kontrollen ausgeführt, die so quasi eine ,Schwangerschaft auf Probe zur Folge haben, und auch die Frage der Kosten von Behinderungen wird unter dem Aspekt der Vermeidbarkeit diskutiert. $^{84}$

Den ,Nutzen ' dieser Erkenntnisse für die interdisziplinäre Geschlechterforschung sehe ich zunächst einmal darin, dass sie darauf aufmerksam machen, welche, Traditionen eine Entwicklung hat. Ihre Langfristigkeit lässt zudem darauf schließen, dass hier zentral Fragen angesprochen werden, die - wenn auch nicht in ihrer Form - so doch in ihrer Funktion immer wieder hergestellt werden. Dazu gehört die Feststellung, dass auch Kontinuität und insbesondere die tatsächliche oder auch nur scheinbare Kontinuität der Geschlechterverhältnisse in historischen Prozessen ,hergestellt' wird. Die Geschichtsschreibung hat mit der Vorstellung, dass es ihre, eigentliche' Aufgabe sei, (Um-)Brüche zu analysieren viel zur ,Unterbewertung' der Bedeutung und Qualität von Kontinuitäten beigetragen. Erst die Analyse von Kontinuitäten ermöglicht nämlich zu sehen, wie viel Macht und Kraft es bedarf, um eine Veränderung zu verhindern und die Kontinuität und Konstanz der (Macht-)Verhältnisse zu erhalten. Mit dieser Analyse lässt sich unter anderem auch biologistischen Erklärungen in Bezug auf die Konstanz weiblicher Existenz begegnen. Gleichzeitig wird die Vorstellung des Bruchs mit der Vergangenheit als Charakteristikum der Moderne in Frage gestellt und unterschiedliche Pfade im Prozess der Entstehung der modernen Gesellschaft sichtbar gemacht.

Die Geschichte der Eugenik verdeutlicht zudem erneut die Funktionen und Konstruktionsmomente von Brüchen und Zäsuren in der Wissenschaftsgeschichte und verweist bei einer Gegenüberstellung der verschiedenen Textsorten auf unerwartete und vieldeutige Kontinuitäten der Vormoderne, der Moderne und der Postmoderne. Gerade bei der Abgrenzung zwischen Eugenik und Genetik nicht nach dem Zuwachs an

83 Alice Schwarzer, unter <http://www.politeia.uni-bonn.de/pdf/schwarzer.pdf>, Zugriff 24.8.2007.

84 Anne Waldschmidt, Normalität, Genetik, Risiko. Pränataldiagnostik als „government by security“, in: Ulrike Bergermann Hg., Techniken der Reproduktion. Medien - Leben - Diskurse, Königstein 2002, $121-144$. 
Erkenntnissen - die der Genetik ja kaum abzustreiten sind - sondern nach dem ,Funktionieren' der Wissenschaft im Kontext der Geschlechterverhältnisse zu fragen, eröffnet neue und vielfältige Interpretationsmöglichkeiten. Sie ermöglicht auch, Nietzsches Historismuskritik als Kritik an der Reproduktion der Machtverhältnisse zu verstehen und Foucaults Interesse an Regeln, Bedingungen und Modalitäten der Ordnungen wichtig zu nehmen - ohne ihre Geschlechterblindheit zu übernehmen.

Die Komplexität einer Entwicklung, die zunächst als Befreiung interpretiert werden kann, in der weiter zurückliegenden Zeit aber alle Elemente elementaren Zwanges birgt, diese in die Gegenwart überführt und für die künftige Entwicklung zumindest Unsicherheit und Ängste auslöst, ${ }^{85}$ zeigt was , loss of the grand teleological narrative of emancipation", wie es Scott formuliert hat, bedeuten kann: Es macht vorsichtig gegenüber den Vorstellungen von ständig voranschreitender Emanzipation und dem Fortschritts-Narrativ, das für sich in Anspruch nimmt, über das ,richtige' Wissen zu verfügen. Es macht aber auch neugierig auf eine Konzeption von Geschichte, die nicht nur als Vorgeschichte zur Gegenwart ihre Bedeutung erlangt.

\section{Fazit}

Zygmunt Bauman geht davon aus, dass der Wille zur Ordnung und die Vorstellung, Ambivalenz ausschalten zu können, die der Moderne inhärent sind, immer wieder neue Ambivalenzen schaffen. Damit ist die Eugenik nicht „Rückfall in die Barbarei“ und Widerspruch zur Moderne, sondern ein „Projekt der Moderne“. ${ }^{86}$ Die äußerst unterschiedlichen Felder der Historiographiegeschichte und der Geschichte der Eugenik zeigen, dass der Eindruck der Einheitlichkeit, Gradlinigkeit und Zielgerichtetheit von Entwicklungen sich nur allenfalls dann aufrecht erhalten lassen, wenn die Frauen und die Entwicklung der Geschlechterverhältnisse unberücksichtigt bleiben und - überspitzt gesagt - die Einheit der Geschlechtertheorie durch die Ausklammerung der historischen Vielfalt zustande kommt. Dies ist keine neue, aber doch wohl eine unterschätzte Erkenntnis der Frauen- und Geschlechtergeschichte, die die ,allgemeine Geschichte und die Geschlechterforschung bisher nicht ausreichend zur Kenntnis genommen haben.

Der von Joan Scott angeführte Verlust des „grand teleological narrative of emancipation" erhält eine durchaus positive Bewertung, wenn man es als zentrales Element der Geschlechterforschung ansieht, Positionen und Bedeutungen wie Fortschritt und Rückschritt oder die „enabling forces“, aber auch Identitäten wie maskulin und feminin, Kategorien wie Modernisierung und Beharrung, Bruch und Kontinuität kritisch zu

85 Jürgen Habermas, Die Zukunft der menschlichen Natur. Auf dem Weg zu einer liberalen Eugenik, Frankfurt a. M. 2005.

86 Regina Wecker, Eugenik - ein Projekt der Moderne?, in: dies. u. a., Eugenik, wie Anm. 72. 
hinterfragen, dann liegt hier Potential der historischen Forschung. Dabei erscheint es mir als die spezifische kritische Kraft der Geschichtswissenschaft, dass sie die Widersprüche historischer Entwicklungen aufzeigt und damit zugleich auf die Widersprüche der Gegenwart aufmerksam macht. Gudrun-Axeli Knapp hat „mehr historische Tiefenschärfe ${ }^{\text {"87 }}$ gefordert im „Projekt“ der Analyse der Moderne. Die Stärkung der historischen Dimension, sei - schreibt sie - notwendig, wenn die Forschung die komplexen Konstellationen der Entstehung der modernen Gesellschaft angemessen deuten soll. Dass ihre Fragestellungen und Forschungsergebnisse Eingang in die Gender Studies finden, ist nun aber umgekehrt auch für die Geschlechtergeschichte von strategischer und inhaltlicher Bedeutung: Dieser Einbezug ermöglicht die Reflexion im Kontext interdisziplinärer Forschung und stärkt gleichzeitig ihre Stellung auch innerhalb der Geschichtswissenschaft. Die Frauen- und Geschlechtergeschichte ist wohl auf diese Visibilität erneut und gerade jetzt angewiesen.

87 Gudrun-Axeli Knapp, „Intersectionality“. Feministische Perspektiven auf Ungleichheit und Differenz im gesellschaftlichen Transformationsprozeß. Manuskript des Vortrags am 30.11.2006 im Rahmen der Ringvorlesung „Freiheit und Geschlecht - Offene Beziehungen, Prekäre Verhältnisse“ an der Universität Wien, unter <www.univie.ac.at/gender/fileadmin/user_upload/gender/abstracts_ringvorlesung/ Knapp.doc>, Zugriff 24.8.2007. 